\title{
$\mu F E$ models can represent microdamaged regions of healthy and metastatically involved whole vertebrae identified through histology and contrast enhanced $\mu \mathrm{CT}$ imaging
}

Choudhari C., Chan K., Akens M. K., and Whyne C. M.

Version Post-Print/Accepted Manuscript

Citation Choudhari C, Chan K, Akens MK, Whyne CM. $\mu$ FE models can

(published version) represent microdamaged regions of healthy and metastatically involved whole vertebrae identified through histology and contrast enhanced $\mu$ CT imaging. J Biomech. 2016 May 3;49(7):110310. doi:

10.1016/j.jbiomech.2016.02.034. PMID: 26947031

Copyright / License

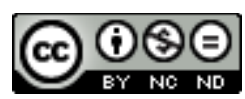

This work is licensed under a Creative Commons Attribution-

NonCommercial-NoDerivatives 4.0 International License.

Publisher's Statement The final published version of this article is available at Elsevier via https://dx.doi.org/10.1016/j.jbiomech.2016.02.034.

Always cite the published version, so the author(s) will receive recognition through services that track citation counts, e.g. Scopus. If you need to cite the page number of the TSpace version (original manuscript or accepted manuscript) because you cannot access the published version, then cite the TSpace version in addition to the published version using the permanent URI (handle) found on the record page. 


\title{
$\mu F E$ models can represent microdamaged regions of healthy and metastatically involved whole vertebrae identified through histology and contrast enhanced $\mu \mathrm{CT}$ imaging.
}

\author{
Chetan Choudhari $^{1,4}$, Katelyn Chan ${ }^{1}$, Margarete K. Akens ${ }^{2,3}$, Cari M. Whyne ${ }^{1,3,4}$. \\ ${ }^{1}$ Sunnybrook Research Institute, Toronto, ON, Canada, ${ }^{2}$ TECHNA Institute, University Health Network, Toronto, \\ ON, Canada, ${ }^{3}$ Department of Surgery, Toronto, ON, Canada, ${ }^{4}$ Institute for Biomaterials and Biomedical \\ Engineering, Toronto, ON, Canada,
}

\begin{abstract}
Microdamage formation within the skeleton is an important stimulant for bone remodelling, however abnormal build-up of microdamage can lead to skeletal fragility. In this study, $\mu \mathrm{CT}$ imaging based micro finite element $(\mu \mathrm{FE})$ models were used to evaluate tissue level damage criteria in whole healthy and metastatically-involved vertebrae. T13-L2 spinal segments were excised from osteolytic $(n=3)$ and healthy $(n=3)$ female athymic rnu/rnu rats. Osteolytic metastasis was generated by intercardiac injection of HELA cancer cells. Micro-mechanical axial loading was applied to the spinal motion segments under $\mu \mathrm{CT}$ imaging. Vertebral samples underwent $\mathrm{BaSO}_{4}$ staining and sequential calcein/fuchsin staining to identify load induced microdamage. $\mu \mathrm{CT}$ imaging was used generate specimen specific $\mu \mathrm{FE}$ models of the healthy and osteolytic whole rat vertebrae. Model boundary conditions were generated through deformable image registration of loaded and unloaded scans. Elevated stresses and strains were detected in regions of microdamage identified through histological and $\mathrm{BaSO}_{4}$ staining within healthy and osteolytic vertebral models, as compared to undamaged regions. Additionally, damaged regions of metastatic vertebrae experienced significantly higher local stresses and strains than those in the damaged regions of healthy specimens. Areas identified by $\mathrm{BaSO}_{4}$ staining, however, yielded lower levels of stress and strain in damaged and undamaged regions of healthy and metastatic vertebrae as compared to fuschin staining. The multimodal (experimental, image-based and computational) techniques used in this study demonstrated the ability of local stresses and strains computed through $\mu \mathrm{FE}$ analysis to identify trabecular microdamage, that can be applied to biomechanical analyses of healthy and diseased whole bones.
\end{abstract}




\section{Introduction}

27 Physiological microdamage formation within the skeleton serves as a stimulant for bone remodeling. Accumulation of unrepaired microdamage in trabecular bone can result from inferior bone quality due to age-related changes or skeletal pathology (Iwata 2014). Despite the importance of microdamage to the mechanical properties of bone, the trabecular stresses and strains experienced at its initiation are not well characterized. Evaluation of stresses and strains associated with microdamage initiation at the local level may enable improvement of fracture risk assessment techniques and the development of therapeutic methods for treatment of skeletal fragility diseases such as metastasis.

Microdamage sites can be experimentally identified through sequential staining and histomorphometry (Lee 2000). Sequential histologic staining (i.e. calcein green and fuchsin) has been utilized to differentiate pre-existing and test induced microdamage (Lee 2000, Herblum 2013). Barium sulfate $\left(\mathrm{BaSO}_{4}\right)$ contrast enhanced $\mu \mathrm{CT}$ imaging has been used as a nondestructive $3 \mathrm{D}$ alternative to conventional histology to detect accumulation of microdamage in trabecular and cortical bone (Landrigan 2011, Wang 2007).

Micro-finite element analysis ( $\mu$ FEA) has been utilized to study local damage initiation properties of trabecular bone (Herblum 2013, Nagaraja 2005). This technique converts segmented $\mu \mathrm{CT}$ voxels representing bone directly into finite elements, yielding large models which can represent complex structural tissue organization. $\mu$ FEA has the capability to model the trabecular morphology, allowing calculation of stresses and strains at histologically identified damage sites (Keaveny 2001). Nagaraja et al. used this approach to determine variations in microdamage initiation parameters in response to age related changes in human and bovine trabecular bone cores (Nagaraja 2005, 2007, 2011, Green 2011). $\mu$ FEA of whole bones allows loading through joints/soft tissues, simulating more physiological loading conditions. Herblum et al. recently demonstrated successful application of $\mu$ FEA to show elevated stresses and strains in regions containing mechanically induced microdamage within whole healthy rat vertebrae

52 (Herblum 2013).

53 Spinal metastasis progressively degrades the trabecular architecture of the vertebral body, leading to an increased risk of fracture (Kurth 2001). Previous studies have demonstrated significant differences in microstructural parameters (i.e. trabecular thickness, trabecular number, etc.) between healthy and metastatically involved vertebrae (Hojjat 2011). The structural parameters do affect the distribution of stresses and strains across the trabecular microarchitecture, but the geometric distribution of bone tissue is not sufficient to explain all the difference (Hojjat 2012). It is not clear if the initiation and propagation of unrepaired microdamage, which precedes fracture, is distinct in metastatic spines. This study aims to generate $\mu \mathrm{FE}$ models that accurately represent damage initiation of whole healthy and osteolytic vertebrae based on histological and contrast enhanced $\mu \mathrm{CT}$ damage quantification and to 63 determine thresholds for damage initiation based on these models. It is hypothesized that $\mu \mathrm{FEA}$ will yield consistent damage initiation thresholds in healthy and osteolytic vertebrae. 
Animal models:

67 The workflow of this study is included as Figure 1. A previously described rat tumor model was

68 used to generate osteolytic metastasis in rat spines through intracardiac injection of HeLa cells

69 (Burch, 2007). Three osteolytic and three healthy rnu/rnu female rats of similar age (7-8 weeks) and weight (160-170g) were sacrificed and T13-L2 spinal motion segments extracted.

\section{Loading and $\mu \mathrm{CT}$ imaging of spinal motion segments:}

A $\mu \mathrm{CT}$ compatible loading device (Figure 2) was used to induce microdamage in the healthy and metastatically-involved spine samples. The loading device allows for $\mu \mathrm{CT}$ scanning of the samples while under load. The top and bottom vertebrae of each 3 level motion segment were potted in a custom jig with PMMA to prevent lateral movement of the sample while loading. The motion segments of the healthy and osteolytic rats were preconditioned under uniaxial compression for 3 cycles at $40 \mathrm{~N}$ at a constant strain rate of $3 \mu \mathrm{m} / \mathrm{s}$. Axial compressive loads of $100 \mathrm{~N}$ and $50 \mathrm{~N}$ were applied to the healthy and metastatic spinal motion segments for 3 hours to create microdamage in the respective samples without fracture (Herblum 2013, Hardisty 2012). $\mu \mathrm{CT}$ images of unloaded and loaded spinal motion segments were acquired at an isotopic voxel size of $11.4 \mu \mathrm{m}$ at $55 \mathrm{KeV}$ and $200 \mu \mathrm{A}$ ( $\mu \mathrm{CT}-100$, Scanco Medical, Brüttisellen, Switzerland).

\section{Specimen staining}

Vertebral samples were immersed in a $1 \%$ calcein solution in distilled water with $0.9 \% \mathrm{NaCl}$ and $2 \% \mathrm{NaHCO}_{3}$ and placed under vacuum for 16 hours to identify pre-existing trabecular damage (Herblum 2011). Following load application, the middle vertebrae (L1) were excised and stained with $\mathrm{BaSO}_{4}$ to identify load induced damage, and reimaged. For $\mathrm{BaSO}_{4}$ staining, the samples were first soaked in a solution of equal parts by volume of distilled water, acetone and $\mathrm{BaCl}_{2} .2 \mathrm{H}_{2} \mathrm{O}$ for 1 day. The samples were then immersed in a solution containing equal parts by volume of PBS, acetone and $\mathrm{Na}_{2} \mathrm{SO}_{4}$ for another day. Each step was followed by 1 hour washes in distilled water to remove excess ions (Landrigan 2011). The samples were also stained with $1 \%$ solution of basic fuchsin hydrochloride through one hour sequential steps in a series of graded ethyl alcohols $(80 \%, 80 \%, 95 \%, 95 \%, 100 \%$ and 100\%) under vacuum (Burr 1995). Pilot studies demonstrated no adverse interactions from the multiple staining protocols. Fuchsin has been used previously in combination with calcein (Herblum 2013) and with $\mathrm{BaSO}_{4}$ (Landrigan 2011). Pilot studies have also shown that $\mathrm{BaSO}_{4}$ may stain certain regions of trabecular bone independent of calcein/fuchsin staining.

\section{$100 \quad$ Histologic slide scanning and $\mu \mathrm{CT}$ imaging}

101 The stained samples were embedded in methylmethacrylate for histology. Coronal sections of $10260-80 \mu \mathrm{m}$ thickness were prepared from each of the embedded samples. The histology slides were 
scanned at 20x magnification using bright field and green filtered fluorescence imaging (Mirax digital slide scanning system)). Locations of linear microcracks stained only with fuchsin were identified to indicate the presence load induced microdamage on individual trabeculae. Damage regions were identified prior to $\mu \mathrm{FE}$ model creation, but blinded to the individual conducting the $\mu$ FEA to prevent bias.

$\mu \mathrm{CT}$ scans of the histology slides were also acquired to validate $\mathrm{BaSO}_{4}$ against histological staining. The presence and absence of $\mathrm{BaSO}_{4}$ in the histologically identified damaged regions selected was examined, and the presence of $\mathrm{BaSO}_{4}$ stain in sites not containing fuchsin or 111 calcein.

\section{Alignment of histology slides}

$114 \mu \mathrm{CT}$ scans were thresholded to segment the bone from surrounding non-bone areas. The 3D 115 segmented $\mu \mathrm{CT}$ images of the histology slides were registered to the respective unloaded $\mu \mathrm{CT}$ 116 scans of the whole samples. Using the histology blocks as a reference point, the slides were first 117 aligned to the unloaded scan manually, followed by automated alignment. Once a slide was 118 aligned, the region within the unloaded $\mu \mathrm{CT}$ dataset corresponding to the hisology slide was 119 identified as a separate material. This allowed for the separation of $\mu \mathrm{FE}$ elements corresponding 120 to the histology slides for alanysis. This technique has been successfully used without affecting FE model continuity (Herblum 2013). The accuracy of the alignment was assessed by calculating the volumetric concurrency (VC) of the two scans. VC was evaluated as the ratio of bone overlap between the histology slide and the unloaded scan to the bone volume of the histology slide. Post alignment, the segmented scans were downsampled to a voxel size of $35 \mu \mathrm{m}$ to reduce the size of the model (Herblum 2013).

To register the locations of $\mathrm{BaSO}_{4}$ staining in the $\mu \mathrm{FE}$ models, the $\mu \mathrm{CT}$ scans of the unloaded and $\mathrm{BaSO}_{4}$ stained vertebrae were utilized. As above, extraction of $\mu \mathrm{FEA}$ results for the elements corresponding to the $\mathrm{BaSO}_{4}$ staining was limited to the area contained in the excised slides, for direct comparison with identified microdamage.

133 The $\mu \mathrm{FE}$ models were generated in AmiraDEV 5.2.2 from the unloaded $\mu \mathrm{CT}$ scans of the middle 134 vertebra, with histology slides defined as separate element sets. A voxel based meshing 135 algorithm was used to generate a mesh of 8-noded hexahedral elements from the segmented $\mu \mathrm{CT}$ 136 scans (Herblum 2013). The mesh was then converted into an Abaqus input file (ABAQUS, 137 Pawtucket, RI). A previously developed deformable image registration algorithm was used to 138 determine boundary conditions for the FE models (Hardisty 2009). Using the image registration 139 of the loaded/unloaded scans, fifty to eighty thousand displacement vectors were extracted for 140 the FE analysis. A custom built algorithm was used to assign these displacement fields as FE 141 boundary conditions at surfaces of the endplates of the vertebral body and the facet joints under 
142 axial compressive load (Herblum 2013, Nagaraja 2005, 2007). Each final Abaqus input file 143 included the mesh with identified histology slides elements, loading surfaces and boundary 144 conditions (Figure 3). A Young's modulus and a Poisson's ratio of $12.5 \mathrm{GPa}$ and 0.3 respectively 145 were assigned as material properties for bone (Kinney 2000). Each of the isotropic, 146 homogeneous, linear elastic models, containing over a million elements, was analyzed using 147 Abaqus Standard 10-1 on the supercomputing facility at SciNet, University of Toronto.

\section{Data analysis}

150 The slices within the $\mu \mathrm{FE}$ models corresponding to the histology slides were isolated for 151 comparison with histology. For quantitative analysis, regions of microdamage highlighted by the 152 fuchsin $(n=20)$ were selected from both groups (healthy and metastatic). Surrounding undamaged

153 regions $(n=20)$ were also selected for comparison (Herblum 2013, Nagaraja 2005, 2007). The 154 finite elements corresponding to these regions were identified within the models. Stress (Von 155 Mises and maximum principal) and strain (maximum principal) parameters from the damaged 156 and undamaged regions from both healthy and osteolytic models were also compared. Normal 157 distribution of this data was verified through One-Sample Kolmogorov-Smirnov Tests. Two-way 158 ANOVA tests were used to compare stress/strain values in the damaged and undamaged regions 159 of the same group, as well as those in osteolytic and healthy bones. A repeated measures linear 160 regression analysis was also run to assess group differences on each continuous measure 161 adjusting for the correlation among observations from the same specimen.

Ten additional microdamage sites stained only by $\mathrm{BaSO}_{4}$ (and not calcein or fuchsin) were selected for both healthy and metastatic models. Similar analysis was performed to compare the stresses and strains in the damaged and undamaged regions. Additional two-way ANOVA tests were performed to compare the local stresses and strains in the damaged regions stained by these

\section{Results}

\section{Histologic microdamage identification and alignment with $\boldsymbol{\mu C T}$}

171 Both calcein and fuchsin stained microdamage in whole healthy and osteolytic vertebrae (Figure

172 4). After loading fuchsin was observed to stain the pre-existing and mechanically induced 173 microdamage. Calcein staining prior to loading only stained a-priori defects. Fuchsin-stained 174 regions not containing calcein were identified as areas damaged by the applied mechanical 175 loading ( $\mathrm{n}=20$ healthy, $\mathrm{n}=20$ metastatic).

$176 \mu \mathrm{CT}$ images of the histology slides were registered to the unloaded $\mu \mathrm{CT}$ scans using manual and 177 rigid affine registration. Alignment of the tissue block containing the posterior elements 178 facilitated registration of the histology slides which were cut approximately parallel to the block, 
The volumetric concurrency (VC) averaged 68\% (range=63-77\%) for slides from healthy samples $(n=7)$ and $64 \%$ (range $=59-79 \%)$ for slides from metastatic samples $(n=8)$.

\section{Stresses and strains in histologically identified damaged and undamaged regions}

183 The elements corresponding to the selected fuchsin stained regions ( $\mathrm{n}=20$ healthy, $\mathrm{n}=20$ 184 metastatic) were identified within the $\mu \mathrm{FE}$ models (Figure 5). Damage initiation thresholds were identified as $53-80 \mathrm{MPa}$ (principal stress) and $0.47-0.71 \%$ (principal strain) in healthy samples. In metastatic bones, the analogous values were found to be $78-127 \mathrm{MPa}$ and $0.66-0.98 \%$. A one sample Kolmogorov-Smirnov (KS) test verified the normal distribution of the stress and strain values. Two-way ANOVA revealed that the von Mises stresses, maximum principal stresses and strains in the damaged regions were significantly greater than those in the undamaged regions for healthy and metastatic models (Tables 1 and 5).

191 Using independent two-way ANOVA tests, the average maximum principal stress and strain 192 were significantly higher in the damaged regions of metastatic vs. healthy vertebrae, with a 193 similar trend for von Mises stress (Tables 2 and 5). Similar findings were observed for damaged 194 (28-58\%) and undamaged (23-47\%) regions. Interactions between the two variable groups were 195 not significant. The repeated measures linear regression analysis demonstrated significant group 196 differences for principal stress and strain adjusting for the correlation among observations from 197 the same specimen $(\mathrm{p}<0.05)$, but the difference was not found to be significant for maximum 198 Von Mises stress $(\mathrm{p}=0.18)$.

\section{Stresses and strains in $\mathrm{BaSO}_{4}$ identified damaged regions}

201 Each of the 40 histologically identified damage sites were compared with the corresponding $\mu \mathrm{CT}$ 202 scans for presence of $\mathrm{BaSO}_{4} .35 / 40$ fuchsin stained damage sites were found to be enhanced by $203 \mathrm{BaSO}_{4}$ (efficiency of 87.5\%). Imperfect segmentation or improper alignment may cause $\mathrm{BaSO}_{4}$ 204 to not be observed in some fuchsin stained regions. Additionally, in some cases calcein and 205 fuchsin stains may stain old features or small proteins masking the $\mathrm{BaSO}_{4}$ staining.

$\mathrm{BaSO}_{4}$ was also found in regions of bone not stained by either calcein or fuchsin. These regions may represent finer microcracks, that only smaller barium and sulfate ions can penetrate (Landrigan 2009). Regions containing only $\mathrm{BaSO}_{4}$ (and not calcein and fuchsin) demonstrated elevated maximum principal stresses and strains and von Mises Stress (metastatic only) in the damaged regions (Tables 3 and 5). Regions of $\mathrm{BaSO}_{4}$ identified microdamage in the metastatic models were found to have higher principal stresses and strains in the damaged and undamaged

212 regions as compared to healthy models, with a similar trend for average Von Mises stresses

213 (Tables 4 and 5). 


\section{Discussion}

216 Reductions in the quality of metastatic bone (lower density, architectural weaknesses) lead to 217 increased occurrence of microdamage. Understanding the initiation and the progression of

218 microdamage to fracture can improve the mechanical assessment of bone. Previous studies in 219 non-pathologic trabecular bone cores and whole bones have demonstrated elevated tissue level 220 stresses and strains in regions of damage (Herblum 2013, Nagaraja 2007). In this study, 221 specimen specific $\mu \mathrm{FE}$ models were applied to evaluate trabecular damage parameters in healthy 222 and metastatically-involved vertebral specimens.

223 Significantly elevated maximum principal stresses and strains were found in the damaged regions as compared to undamaged ones. Damage initiation was considered to occur at stress/strain values between those found in the undamaged and damaged regions. Trabecular microdamage for healthy rnu/rnu vertebrae initiated between stress and strains in the range of 53$80 \mathrm{MPa}$ and $0.47-0.71 \%$ respectively. Using a similar methodology on larger whole wistar rat vertebrae, Herblum et al. reported damage initiation at $85-128 \mathrm{MPa}$ and $0.9-1.4 \%$ (Herblum 2013). Nagaraja et al. found damage initiated between $92-132 \mathrm{MPa}$ and $0.82-1.21 \%$ in young bovine trabecular cores, and between $81-110 \mathrm{MPa}$ and $0.38-0.64 \%$ in older bovine trabecular sections (Nagaraja 2011). The alterations in local mechanical properties were attributed to age related mineral and architectural changes in trabecular bone. Green et al. revealed that microdamage in human trabecular bone cores initiated in the range of $53-83 \mathrm{MPa}$ and $0.48-0.81 \%$ for young donors, with no significant differences in local damage initiation thresholds for older specimens (50-84MPa and 0.51-0.83\%) (Green 2011). From these studies large intra and interspecimen diversity in local damage initiation properties exist, which may be due to both physiological and structural sample differences.

As in healthy samples, damaged regions exhibited significantly higher maximum principal stresses and strains in metastatic vertebrae. Damage in osteolytic specimens initiated between stress and strain range of $78-127 \mathrm{MPa}$ and $0.66-0.98 \%$ respectively. Osteolytic samples have lower trabecular number and thicknesses (Hojjat 2011). This implies that at similar continuum strains, individual trabecular struts in osteolytic spines have higher stresses compared to healthy bone. Also, as the metastatic spine is structurally weaker, it is closer to bulk yield when loaded under similar strain. Damaged regions have been shown to experience higher stresses and strains when loaded to strains closer to the yield strain (Nagaraja 2005). Herblum reported microdamage initiation in a mixed osteolytic/osteoblastic metastatic model in an rnu/rnu rat vertebra at 224447MPa and 2.5-4.4\% (Herblum 2011) - much higher than those observed in this study. However, local bone mechanics vary significantly with the type of tumor, giving rise to differences in failure initiation properties. Compared with healthy samples, trabeculae in the osteolytic vertebrae experience significantly higher stresses (127MPa vs. $80 \mathrm{MPa}$ ) and strains

251 (0.98\% vs. $0.71 \%)$ in the damaged regions, despite the reduced applied axial load. risk. This study demonstrates a relationship between tissue level stresses and strains with local 
failure events. The quantitative results indicate the stress and strain values as determined for damaged bone vs. undamaged bone, with damage initiation beginning somewhere between the damaged and undamaged range. As such, this study did not aim to predict the stress/strain values at which microdamage initiation occurs; but rather define a range of values where damage may begin. Thus, the models generated in this study were not predictive. The selection of microdamage sites in the healthy and metastatic slides was also not exhaustive, but represented a subset of overall microdamage within the samples since not all microdamage sites on the histology slides could be well visualized. Future work may establish the cut-off stress/strain values for microdamage initiation using $\mu \mathrm{FE}$ models that evaluate the total volumetric presence of microdamage. A few regions, especially near the endplates, that were observed to have stress concentrations without failure (figure 6), may indicate structural diversities across the trabecular mesh could lead to different failure mechanisms and thresholds.

Vertebral $\mu \mathrm{FE}$ models generated at a $35 \mu \mathrm{m}$ voxel size have previously demonstrated sufficient convergence (Neibur 1999, Ladd 1998). At this resolution, each trabecula had at least two voxels in one plane, and more than 4 voxels spanning its thickness. No singularities were observed in the model. The large size of the $\mu \mathrm{FE}$ models and associated computational requirements were the limiting factors for the voxel size utilized. The trabecular network near the growth plates was not well resolved at this resolution, limiting the evaluation of microdamage within these regions. Voxel size also limits the resolution of the mechanical parameters in the damaged areas which may lead to underestimation of the stresses and strains experienced. $\mu \mathrm{FE}$ models at higher resolutions have the potential to address these issues, leading to improved damage initiation criteria. Selectively increasing the resolution of critical regions of the models may represent a future approach to limit computational costs (Herblum 2013).

More advanced non-linear $\mu$ FEA may improve the fidelity of the models by accounting for material softening during damage initiation and stress redistribution to surrounding undamaged elements (Nagaraja 2007). In this study homogeneous material properties were used for both healthy and metastatically involved bone. Metastasis has been shown to alter bone tissue material properties resulting in reduced elastic moduli as compared to normal bone (Nazrin 2008). The heterogeneity of these changes, however, has not been well characterized. Intensity based specimen specific inhomogeneous material property assignment has been shown to yield slightly more accurate local stresses and strains in $\mu$ FE models (Nagaraja 2007). However, implementing this is complicated by potential differences in the quality of healthy and metastatically involved bone, which may not be captured by BMD alone. Using a constant modulus of elasticity in healthy and metastatic bone may have led to overestimation of local stresses and strains in the metastatic models. Localized nanoindentation data from healthy and metastatic vertebrae may ultimately provide data for heterogenous voxel based BMD based mechanical property assignment (Wolfram 2010).

291 A sequential staining routine previously developed and optimized was used to identify regions of 292 pre and post loading microdamage. Calcein acts as a site specific dye for microdamage detection, 
as it chelates and combines with exposed calcium ions on microdamaged surfaces (Lee 2000). $1 \%$ fuchsin staining with graded alcohols was used to identify de novo damage in response to uniaxial compressive loading (Burr 1995). Fuchsin labels microdamage partially by diffusing and accumulating in the damaged region and partially by binding to exposed collagen (Lee 2000). This study extended the sequential stains to evaluate the effectiveness of $\mathrm{BaSO}_{4}$. Fuchsin and calcein stains have a much bigger molecules size compared to $\mathrm{Ba}$ and $\mathrm{SO}_{4}$ ions. As a result, $\mathrm{BaSO}_{4}$ can enter smaller cracks that fuchsin and calcein cannot penetrate (Landrigan 2009). Also $\mathrm{Ba}$ ions may be preferential towards high surface energies and binding sites on the cracked mineral. A strong agreement was detected between regions of microdamage identified by $\mathrm{BaSO}_{4}$ and fuchsin (87.5\%) suggesting that the staining techniques can be used concurrently. The lack of complete concurrency may be due in part to non-ideal registration of the slides to the $\mu \mathrm{CT}$ scans of the whole vertebrae. $\mathrm{BaSO}_{4}$ also labelled calcein stained pre-existing microdamage, and thus was not specific to load induced damage. Sequential staining with $\mathrm{BaSO}_{4}$ could overcome this challenge. To differentiate between pre-existing and newly formed cracks with $\mathrm{BaSO}_{4}$ sequential staining and microCT imaging pre and post loading could be used in future studies. In this manner the pre-existing damage can be quantified in an initial scan and, with accurate alignment, identified in the second (post loading) scan. Additional areas of $\mathrm{BaSO}_{4}$ stain can then be said to represent load induced microdamage (Landrigan 2009). Overall, successful implementation of $\mathrm{BaSO}_{4}$ staining in whole healthy and osteolytic vertebrae represents a significant step forward to establish this technique as a powerful 3D volumetric complement to microscopic methodologies.

$\mathrm{BaSO}_{4}$ was found to stain some regions of bone unstained by calcein or fuchsin. $\mu \mathrm{FE}$ results for such regions from the healthy and metastatic models, were similar to the results for histologically identified microdamage. In comparing stresses and strains in the regions identified by histology and $\mathrm{BaSO}_{4}$, the stresses and strains were consistently lower in $\mathrm{BaSO}_{4}$ only enhanced damage sites, but these differences were not significant. These regions may represent finer microcracks and/or diffuse damage unstained by both calcein and fuchsin. While only linear microcracks were selected for the histologic analysis, $\mathrm{BaSO}_{4}$ staining does not distinguish between linear and diffuse damage at the resolution used. Nagaraja et al. demonstrated that more severe regions of damage yield higher stresses and strains within the $\mu \mathrm{FE}$ model (Nagaraja 2005). As such, the calcein/fuchsin stained regions of microdamage may represent more severe form of damage and consequently yield slightly higher stresses and strains.

325 Physiologic loading was simulated in this study through uniaxial compression applied through intervertebral discs. Multi-axial loading (compression, torsion and bending) may allow further investigation of trabecular failure (including bending and buckling) that in combination with fatigue protocols could provide a more comprehensive description of local failure. In vivo vertebrae are subjected to multi-axial repetitive loading, thus studying their effects on trabecular failure may lead to a more complete understanding of physiological microdamage initiation. 
In the current study, $\mu \mathrm{CT}$ imaging of thick histology slides allowed for volumetric registration between the histology slides and unloaded $\mu \mathrm{CT}$ scans. Thick bone sections facilitated automated rigid volumetric registration for improved efficiency of the alignment process. The alignment enabled identification of identical trabeculae on the slides and unloaded scans. The volumetric concurrencies achieved were acceptable (Herblum 2013), but may have been somewhat limited due to histological processing artefacts (i.e. variable thicknesses). The efficiency of alignment may also have suffered in the metastatic samples as a result of decreased osteolytic bone content. Accurate registration of the histology slides to the unloaded $\mu \mathrm{CT}$ images of whole vertebrae represented an important component of the experimental procedure. This allowed direct comparison of histologically identified load induced microdamage with the $\mu \mathrm{FEA}$ results. Registration of the slide to the unloaded scans also allowed for verification of $\mathrm{BaSO}_{4}$ staining against the gold standard histological staining.

Overall, specimen specific $\mu \mathrm{FE}$ models of healthy and metastatic (osteolytic) vertebrae were successfully generated with accurate integration of histologically defined regions based on volumetric image registration and displacement boundary conditions based on loaded/unloaded $\mu \mathrm{CT}$ image registration. Microdamage was histologically identified through a whole bone sequential calcein/fuchsin labelling technique. The ability of $\mathrm{BaSO}_{4}$ contrast agent to highlight regions of vertebral microdamage was verified against histological staining. In the $\mu \mathrm{FE}$ models, significantly higher stresses and strains were recorded in regions of damage identified by staining. This advanced modelling technique provides a new robust method for validating the ability of $\mu \mathrm{FE}$ modeling to quantify microdamage in skeletal structures, with potential for application to the analysis of healthy or pathologic whole bones under complex physiologic loading conditions.

\section{Acknowledgements}

356 Microdamage: This study was supported by the Canadian Institute of Health Research (Funding 357 reference numbers: 68911 and 125886). The authors thank the University of Toronto Dentistry 358 histology lab and the Imaging Facility at SickKids hospital for histologic sectioning and imaging 359 and Dr A Kiss for statistical assistance.

\section{Conflict of Interest Statement}

362 The authors have no conflict of interest. 
References:

364

Burch S, Bisland SK, Wilson BC, Whyne C, Yee AJ., 2007. Multimodality imaging for vertebral metastases in a rat osteolytic model. Clin Orthop Relat Res. Jan;454:230-6.

Burr, D. B., Hooser, M., 1995. Alterations to the en bloc basic fuchsin staining protocol for the demonstration of microdamage produced in vivo. Bone 17, 431-433.

Evans, S.P., Parr, W.C.H., Clausen, P.D., Jones A., Wroe, S., 2012. Finite element analysis of a micromechanical model of bone and a new 3D approach to validation. Journal of Biomechanics 45, 2702-2705

Green, J., Nagaraja, S., Diab, T., Vidakovic, B. Guldberg, R.E., 2011. Age-related changes in human trabecular bone: Relationship between microstructural stress and strain and damage morphology. Journal of Biomechanics 44, 2279-2285

Hardisty, M.R., Whyne, C.M., 2009. Whole bone strain quantification by image registration: a validation study. Journal of Biomechanical Engineering 131, 064502.

Hardisty, M.R., Akens, M.K., Hojjat, S.P., Yee, A., Whyne, C.M., 2012. Quantification of the Effect of Osteolytic Metastases on Bone Strain within Whole Vertebrae Using Image Registration. Journal of Orthopedic Research 30, 1032-1039.

Herblum, R., 2011. Evaluation of micro level structural integrity of the spine through micro finite element modeling and histological analysis. PhD. Thesis. University of Toronto, Canada

Herblum, R., Beek, M., Whyne, C.M., 2013. $\mu$ FEA successfully exhibits higher stresses and strains in microdamaged regions of whole vertebrae. Journal of Orthopaedic Research 31, 1653-1660.

Hojjat, S.P., Whyne, C.M., 2011. Automated quantitative microstructural analysis of metastatically involved vertebrae: Effects of stereologic model and spatial resolution. Medical Engineering \& Physics 33, 188-194

Hojjat SP, Beek M, Akens MK, Whyne CM., 2012. Can micro-imaging based analysis methods quantify structural integrity of rat vertebrae with and without metastatic involvement? Journal of Biomechanics. Sep 21;45(14):2342-8.

Iwata, K., Mashiba, T., Hitora, T.,Yamagami, Y., Yamamoto, T., 2014. A large amount of microdamages in the cortical bone around fracture site in a patient of atypical femoral fracture after long-term bisphosphonate therapy. Bone 64183-186.

Keaveny, T.M., Morgan, E.F., Niebur G.L., Yeh O.C., 2001. Biomechanics of trabecular bone. Annual Review of Biomedical Engineering 3, 307-333.

Kinney, J. H. et al., 2000. Three-dimensional morphometry of the L6 vertebra in the ovariectomized rat model of osteoporosis: biomechanical implications. Journal of Bone and Mineral Research 15, 1981-1991.

Kurth A.A., Muller, R., 2001. The effect of an osteolytic tumor on the three-dimensional trabecular bone morphology in an animal model. Skeletal Radiology 30, 94-98 
Landrigan, M.D., Li, J., Turnbull, T.L.,Burr, D.B., Niebur, G.L., Roeder, R.K., 2011. Contrastenhanced micro-computed tomography of fatigue microdamage accumulation in human cortical bone. Bone 48, 443-450.

Landrigan, M.D., 2009. Evaluation of methods for measuring microdamage accumulation in cortical bone. PhD Thesis. University of Notre Dame, US.

Ladd, A. J., Kinney, J. H., Haupt, D. L. \& Goldstein, S. A., 1998. Finite-element modeling of trabecular bone: comparison with mechanical testing and determination of tissue modulus. Journal of Orthopedic Research 16, 622-628.

Lee T. C., Arthur T. L., Gibson L. J., Hayes W. C., 2000. Sequential labelling of microdamage in bone using chelating agents. Journal of Orthopedic Research 18, 322-325.

Nagaraja, S., Couse, T. L., Guldberg, R. E., 2005. Trabecular bone microdamage and microstructural stresses under uniaxial compression. Journal of Biomechanics 38, 707716.

Nagaraja, S., Lin, A. S., Guldberg, R. E., 2007. Age-related changes in trabecular bone microdamage initiation. Bone 40, 973-980.

Nagaraja, S., Skrinjar, O., Guldberg, R.E., 2011. Spatial Correlations of Trabecular Bone Microdamage with Local Stresses and strains Using Rigid Image Registration. Journal of Biomechanical Engineering 133, 064502.

Nazarian, A., von Stechow, D., Zurakowski, D., Müller, R., Snyder, B.D., 2008. Bone volume fraction explains the variation in strength and stiffness of cancellous bone affected by metastatic cancer and osteoporosis. Calcifies Tissue International 83(6), 368-79.

Niebur, G. L., Yuen, J. C., Hsia, A. C., Keaveny, T. M., 1999. Convergence behavior of highresolution finite element models of trabecular bone. Journal of Biomechanical Engineering 121, 629-635.

Wang, X., Masse, D.B., Leng, H., Hess, K.P., Ross, R.D., Roeder, R.K., Niebur, G.L., 2007. Detection of trabecular bone microdamage by micro-computed tomography. Journal of Biomechanics 40, 3397-3403.

Wolfram, U., Wilke, H.J., Zysset, P.K., 2010. Valid micro finite element models of vertebral trabecular bone can be obtained using tissue properties measured with nanoindentation under wet conditions Journal of Biomechanics 43(9), 1731-7. 
Figure 1: Experimental design

Figure 2: Loading chamber used to apply axial compressive loading to the spinal motion segments within the $\mu \mathrm{CT}$ scanner (Scanco $\mu \mathrm{CT}$-100).

Figure 3: $\mu \mathrm{FE}$ grid generated form whole bone $\mu \mathrm{CT}$ scan of a metastatic sample with elements corresponding to a histology slide highlighted in grey. One such model was generated from each histology slide

Figure 4: a) Pre-existing damage labelled by both calcein and fuchsin b) Load induced microdamage stained only by fuchsin, and not calcein

Figure 5: a) Axial compressive load induced microdamage identified by fuchsin staining. b) Overlay of the $\mathrm{BaSO}_{4}$ on unstained $\mu \mathrm{CT}$ scan shows the same microdamage site identified using $\mathrm{BaSO}_{4}$ staining. c) Elements corresponding to the damaged site selected in the undeformed $\mu \mathrm{FE}$ model. Elements corresponding to the microdamage site are highlighted in red. d) Completed $\mu$ FEA demonstrates elevated maximum principal stress in the region of microdamage

Figure 6: a) Bright field image of a coronal histology slide from a healthy sample stained with fuchsin to identify load induced microdamage b) Results showing tissue level maximum principal stress distribution in the elements within the $\mu \mathrm{FE}$ model corresponding to the histology slide. Arrows show correspondence between the histology slide and the model. 
Figures

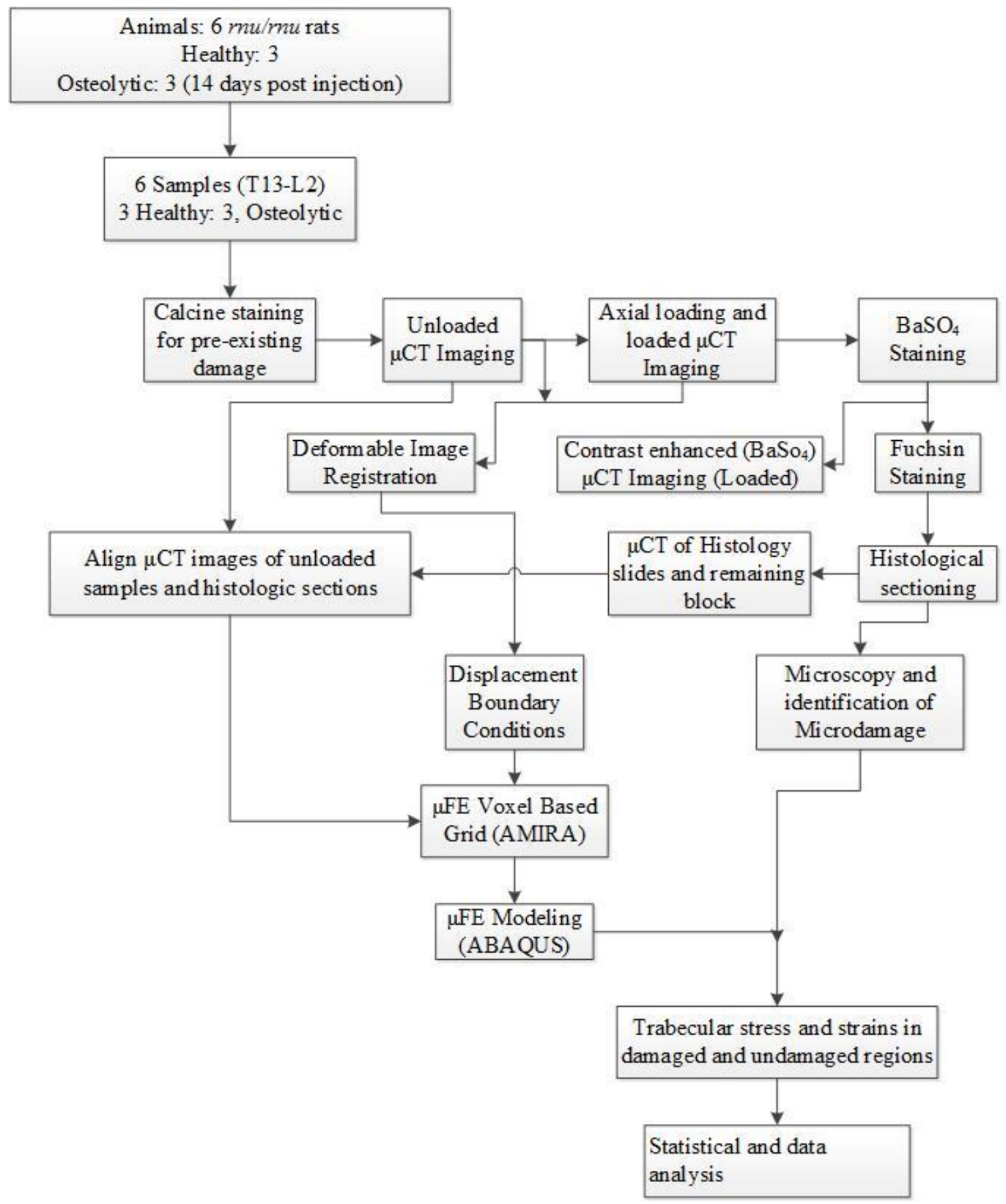

Figure 1 


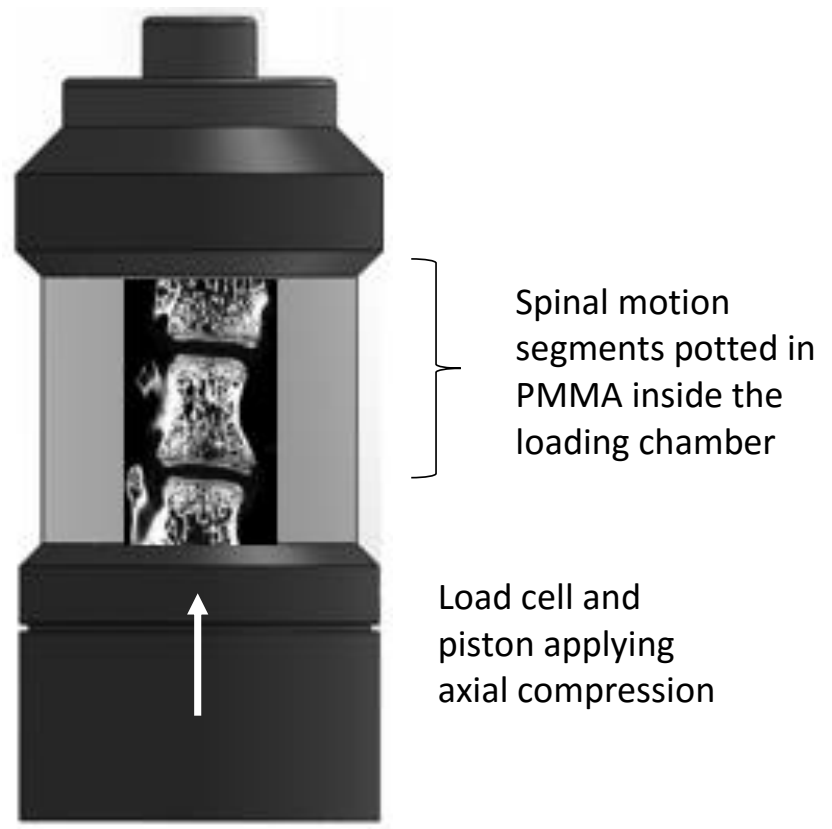

Figure 2 


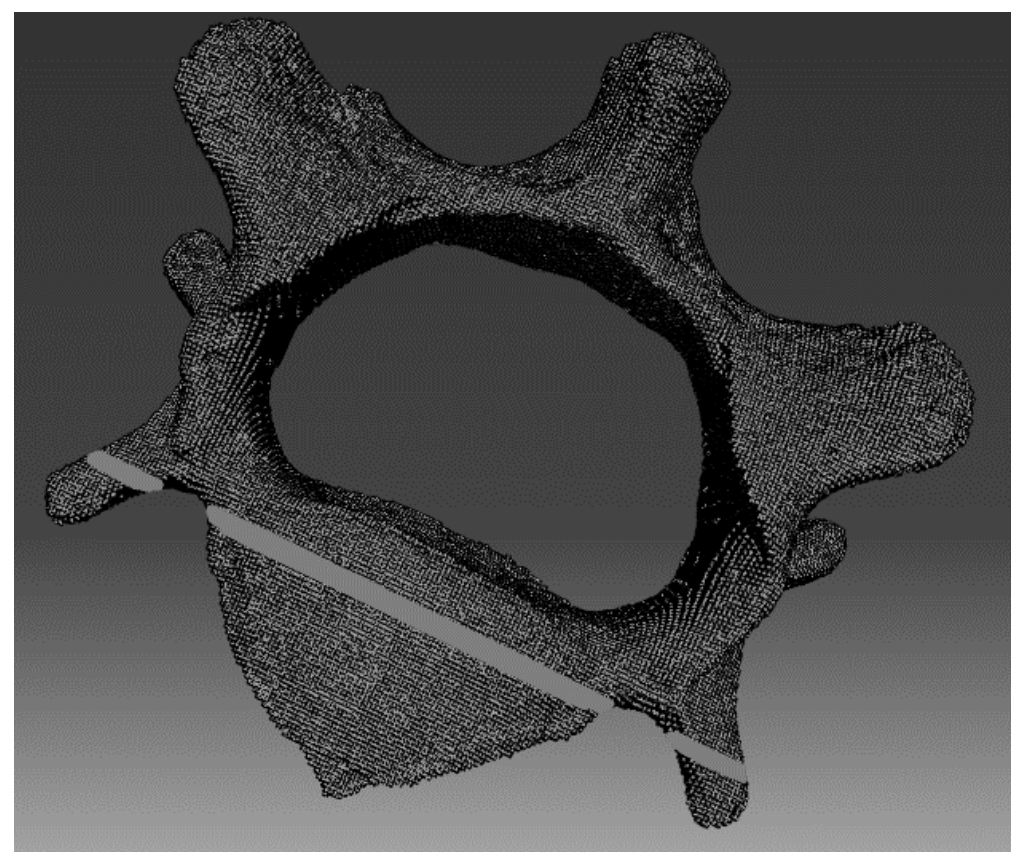

Figure 3 
a)

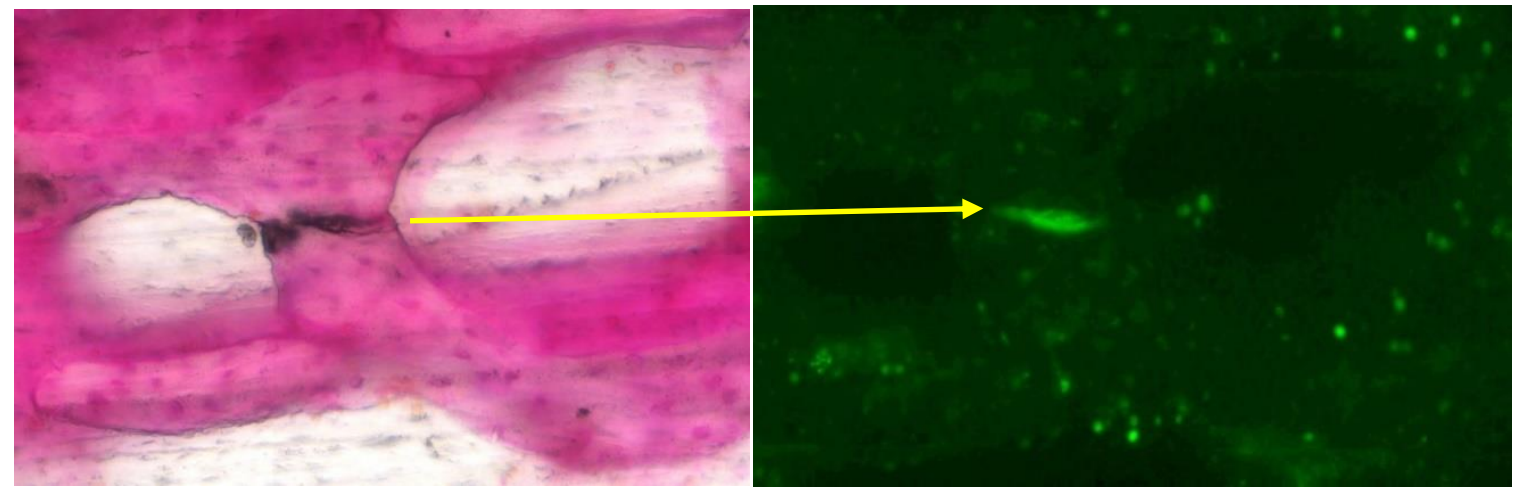

b)

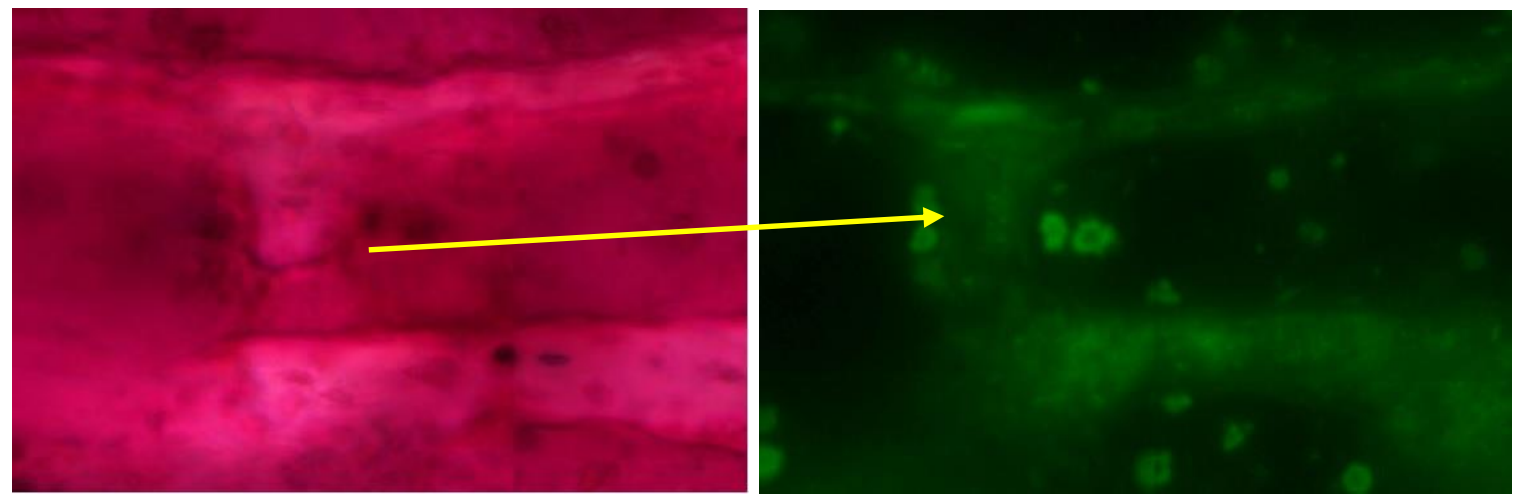

Figure 4 


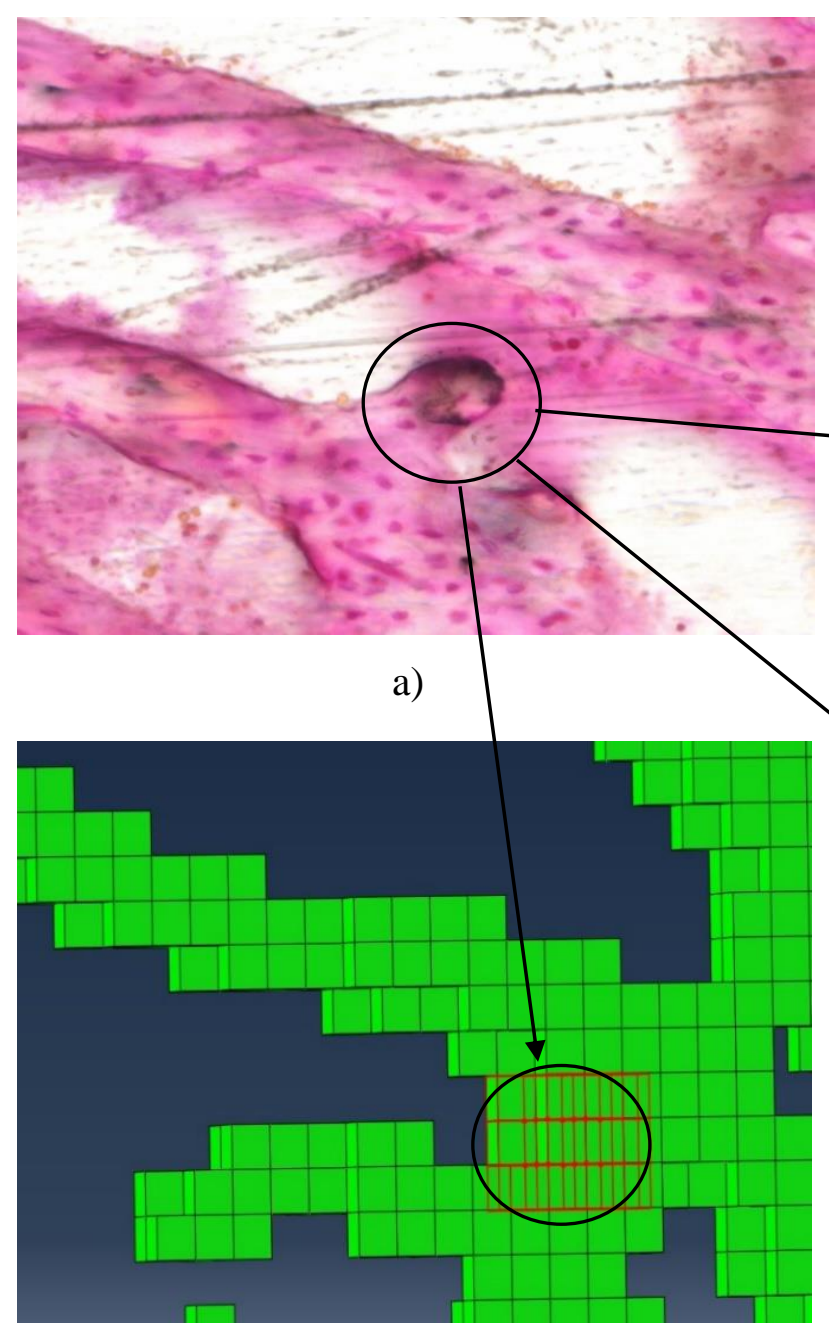

c)

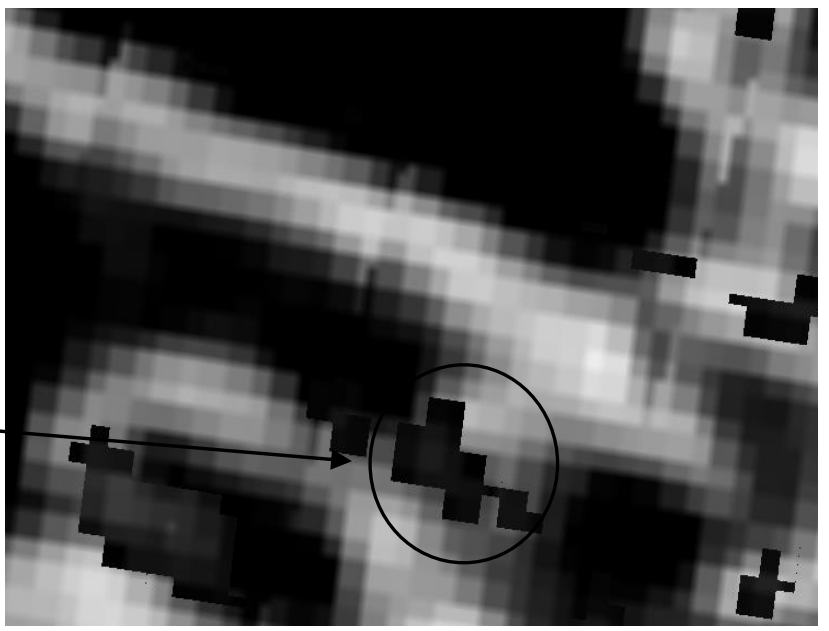

b)

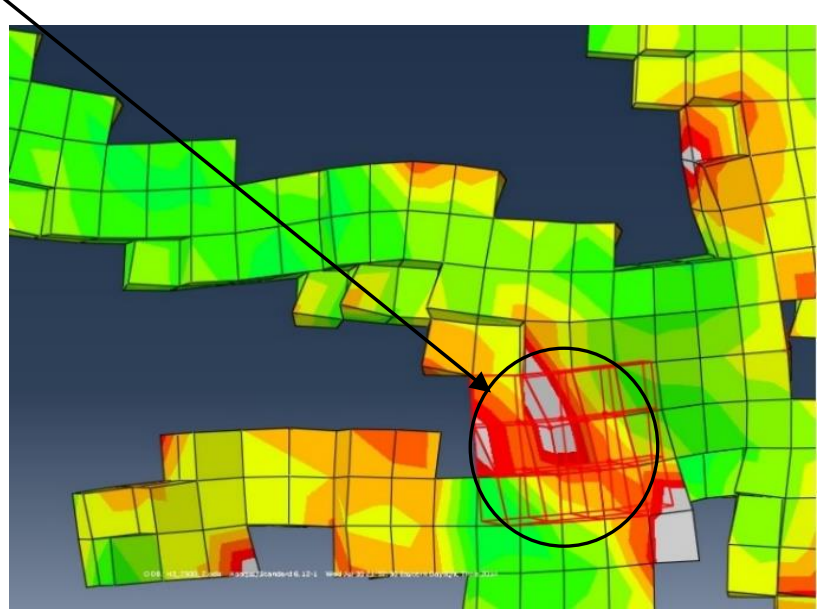

d)

Figure 5 


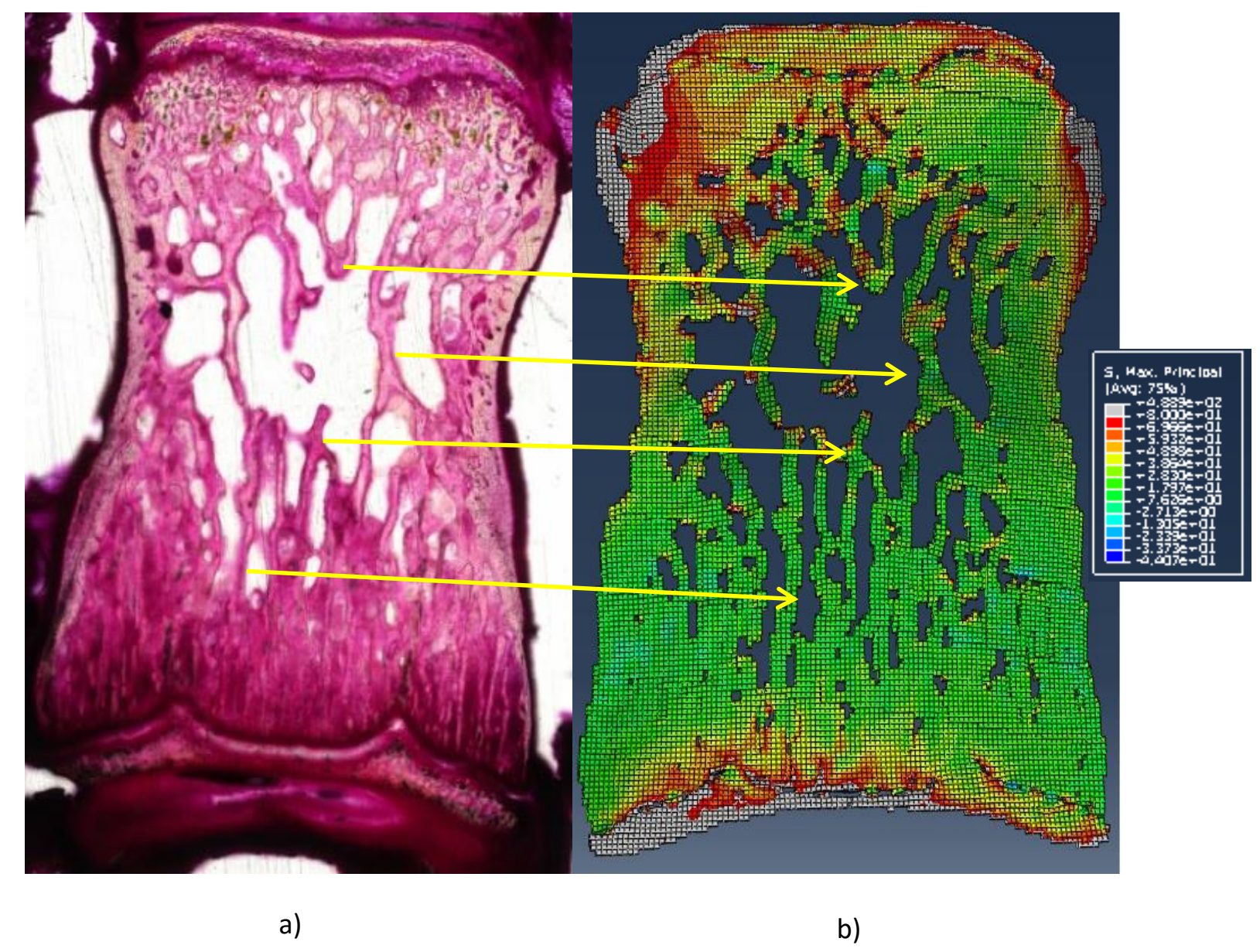

Figure 6 
Table 1: Comparison of local stresses and strains in damaged and undamaged regions $(n=20)$ identified using calcein and fuchsin staining

\begin{tabular}{|c|c|c|c|}
\hline \multirow{3}{*}{ Metastatic } & Damaged & Undamaged \\
\cline { 2 - 4 } & Max Principal Stress (MPa) & $127 \pm 61$ & $108 \pm 35$ \\
\cline { 2 - 4 } & Max Principal Strain (\%) & $0.98 \pm 0.44$ & $78 \pm 32$ \\
\hline \multirow{3}{*}{ Healthy } & Von Mises & $122 \pm 32$ & $0.66 \pm 0.25$ \\
\cline { 2 - 4 } & Max Principal Stress (MPa) & $80 \pm 27$ & $53 \pm 30$ \\
\cline { 2 - 4 } & Max Principal Strain (\%) & $0.71 \pm 0.17$ & $0.47 \pm 0.11$ \\
\hline
\end{tabular}

Table 2: Comparison of local stresses and strains observed in damaged regions $(n=20)$ in healthy and osteolytic models, identified using calcein and fuchsin staining

\begin{tabular}{|c|c|c|c|}
\hline \multirow{3}{*}{ Damaged } & Von Mises (MPa) & Metastatic & Healthy \\
\cline { 2 - 4 } & Max Principal Stress (MPa) & $156 \pm 96$ & $122 \pm 32$ \\
\cline { 2 - 4 } & Max Principal Strain (\%) & $0.98 \pm 0.44$ & $80 \pm 27$ \\
\hline \multirow{3}{*}{ Undamaged } & Von Mises & $108 \pm 35$ & $0.71 \pm 0.17$ \\
\cline { 2 - 4 } & Max Principal Stress (MPa) & $78 \pm 32$ & $88 \pm 30$ \\
\cline { 2 - 4 } & Max Principal Strain (\%) & $0.66 \pm 0.25$ & $0.47 \pm 0.11$ \\
\hline
\end{tabular}

Table 3: Comparison of local stresses and strains in damaged and undamaged regions $(\mathrm{n}=10)$ identified using $\mathrm{BaSO}_{4}$ staining

\begin{tabular}{|c|c|c|c|}
\hline \multirow{3}{*}{ Metastatic } & Damaged & Undamaged \\
\cline { 2 - 4 } & Max Principal Stress (MPa) & $136 \pm 84$ & $92 \pm 36$ \\
\cline { 2 - 4 } & Max Principal Strain (\%) & $0.86 \pm 0.25 \%$ & $65 \pm 17$ \\
\hline \multirow{3}{*}{ Healthy } & Von Mises & $95 \pm 35$ & $0.57 \pm 0.14 \%$ \\
\cline { 2 - 4 } & Max Principal Stress (MPa) & $70 \pm 26$ & $79 \pm 28$ \\
\cline { 2 - 4 } & Max Principal Strain (\%) & $0.58 \pm 0.19 \%$ & $42 \pm 21$ \\
\hline
\end{tabular}

\begin{tabular}{|c|c|c|c|}
\hline \multirow{4}{*}{$\begin{array}{c}\text { Table 4: Comparison of local stresses and strains observed in damaged regions } \\
(\mathbf{n}=\mathbf{1 0}) \text { in healthy and osteolytic models, identified using } \mathrm{BaSO}_{\mathbf{4}} \text { staining }\end{array}$} \\
\hline \multirow{3}{*}{ Damaged } & Von Mises (MPa) & Metastatic & Healthy \\
\cline { 2 - 4 } & Max Principal Stress (MPa) & $136 \pm 84$ & $95 \pm 35$ \\
\cline { 2 - 4 } & Max Principal Strain (\%) & $0.86 \pm 0.25 \%$ & $70 \pm 26$ \\
\hline \multirow{3}{*}{ Undamaged } & Von Mises & $92 \pm 36$ & $0.58 \pm 0.19 \%$ \\
\cline { 2 - 4 } & Max Principal Stress (MPa) & $65 \pm 17$ & $79 \pm 28$ \\
\cline { 2 - 4 } & Max Principal Strain (\%) & $0.57 \pm 0.14 \%$ & $0.40 \pm 0.16 \%$ \\
\hline
\end{tabular}


Table 5: Summary of two way ANOVA

\begin{tabular}{|c|c|c|c|}
\hline & $\begin{array}{c}\text { Damaged vs } \\
\text { Undamaged } p \text {-value }\end{array}$ & $\begin{array}{c}\text { Healthy vs } \\
\text { Metastatic p-value }\end{array}$ & Interaction $\mathrm{p}$-value \\
\hline Von Mises (Histology) & 0.002 & 0.03 & 0.6 \\
\hline Max Principal Stress (Histology) & 0.00002 & 0.00006 & 0.2 \\
\hline Max Principal Strain (Histology) & 0.00002 & 0.00005 & 0.5 \\
\hline Von Mises $\left(\mathrm{BaSO}_{4}\right)$ & 0.1 & 0.07 & 0.4 \\
\hline Max Principal Stress $\left(\mathrm{BaSO}_{4}\right)$ & 0.0002 & 0.001 & 0.6 \\
\hline Max Principal Strain $\left(\mathrm{BaSO}_{4}\right)$ & 0.0005 & 0.0004 & 0.3 \\
\hline
\end{tabular}

